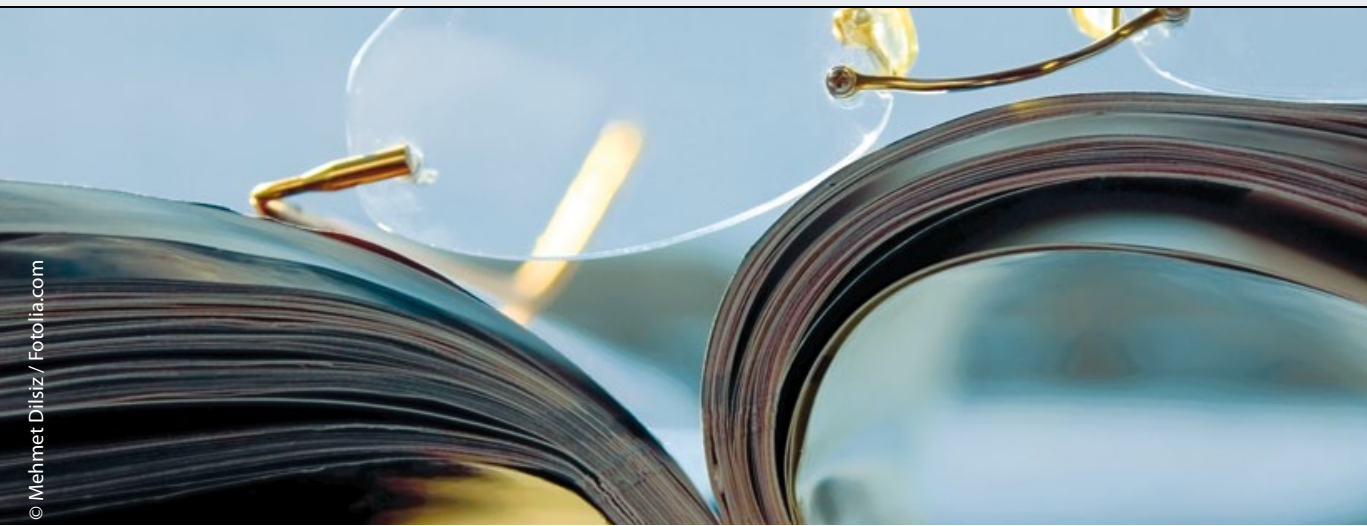

\section{Nicht mehr Zweittumoren bei Androgenentzug}

Die Androgenentzugstherapie beim Prostatakarzinom wird immer wieder mit primären Zweittumoren in Zusammenhang gebracht, etwa mit einem kolorektalen Karzinom. Jetzt sollte eine neue US-Studie die grundsätzliche Assoziation mit Zweittumoren klären helfen.

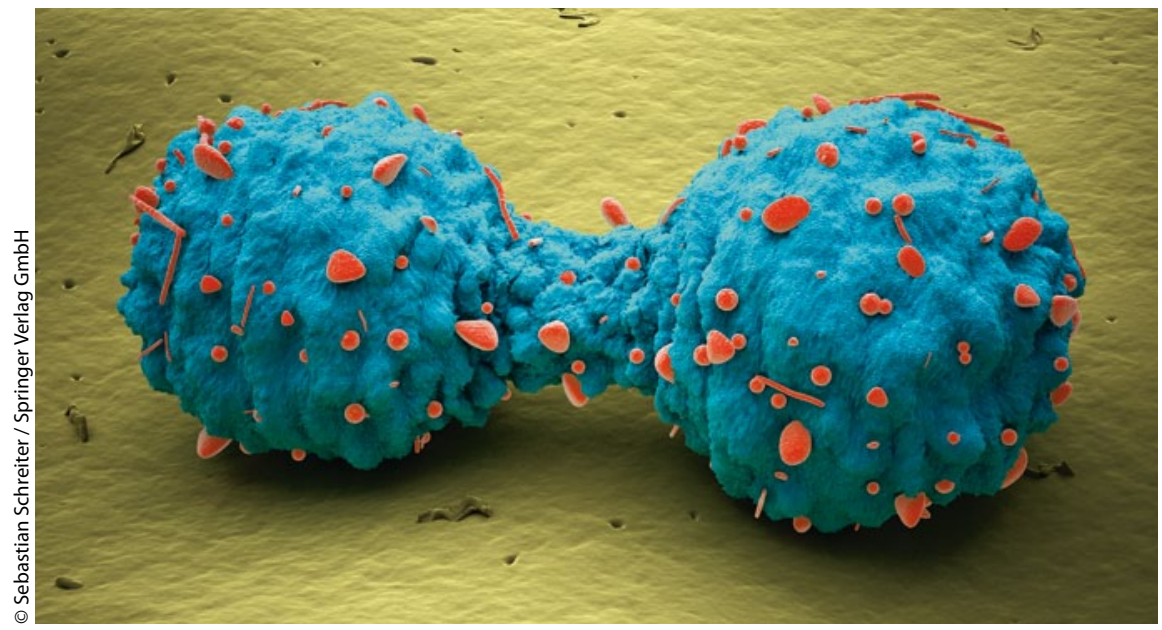

Durch Androgendeprivation steigt das Risiko für Zweittumoren wie Lungenkrebs nicht.

$\mathrm{D}$ er Langzeiteffekt des Androgenentzugs ist bisher nicht eindeutig geklärt. Bisherige Studien haben einen $\mathrm{Zu}$ sammenhang der Hormontherapie mit einem erhöhten Risiko für Osteoporose, Knochenbrüche und kardiovaskuläre Erkrankungen zu Tage gefördert. Auch gibt es Hinweise für eine erhöhte Anfälligkeit der so therapierten Männer für einen primären Zweittumor im Darm. Bekannt ist zudem das erhöhte Risiko für Hyperinsulinämie, Diabetes mellitus und Adipositas.

Im Herbst 2010 hatten US-amerikanische Ärzte in einer Studie mit fast 110.000 Männern des SEER-Registers
(Surveillance, Epidemiology, and End Results) einen Zusammenhang zwischen der Hormontherapie und der Entstehung eines kolorektalen Karzinoms festgestellt [Gillessen S et al. J Natl Cancer Inst 2010;102: 1760-70]. In der Studie stieg das Risiko für Darmkrebs mit der Dauer der Androgenentzugstherapie.

Um dem Zusammenhang mit Zweittumoren auf den Grund zu gehen, wurden nun SEER-Daten von Männern analysiert, bei denen zwischen 1998 und 2007 ein lokalisiertes Prostatakarzinom diagnostiziert worden war. Das Follow-up endete 2009. Die Daten von insgesamt 24.034 Männern, die zum Zeitpunkt der Diagno- se mindestens 18 Jahre alt waren, wurden in der retrospektiven Studie berücksichtigt.

Bei 1.359 Männern (5,7\%) mit lokal begrenztem Prostatakarzinom entwickelten sich Zweittumoren, darunter 187 Kolorektalkarzinome, 178 Lungentumoren, 132 Blasentumoren und 76 Nierenkarzinome. Die betroffenen Männer waren im Vergleich zu jenen, die ausschließlich ein Prostatakarzinom hatten, älter und hatten ein größeres Risiko für Typ2-Diabetes oder Bluthochdruck. Nach Berücksichtigung unter anderem von Alter, ethnischer Zugehörigkeit, Jahr der Krebsdiagnose, Tumorstadium, GleasonScore und Radiotherapie konnten die Wissenschaftler beim Vergleich der Gruppe der Patienten mit Hormontherapie mit jenen ohne Androgenentzug keinen statistisch signifikanten Unterschied feststellen. Auch Diabetes und Adipositas hätten keinen Einfluss auf den Zusammenhang zwischen Zweittumor und Hormontherapie gehabt.

Fazit: Männer mit einem lokal begrenzten Prostatakarzinom, die eine Therapie mit einem GnRH-Agonisten zum Androgenentzug erhalten, müssen wohl nicht befürchten, an einem weiteren Primärtumor, etwa an Lungen- oder Darmkrebs, zu erkranken. Der 2010 beobachtete Zusammenhang mit der Entwicklung von Kolorektalkarzinomen unter der Hormontherapie ließ sich nicht bestätigen. Peter Leiner

Wallner LP et al. Cancer and Risk of Second Primary Malignancies Androgen Deprivation Therapy for Treatment of Localized Prostate Cancer and Risk of Second Primary Malignancies. Cancer Epidemiol Biomarkers Prev 2013; 22: 313-6 